\title{
Identification, subtyping, and tracking of dairy spoilage-associated Pseudomonas by sequencing the iles gene
}

\author{
S. J. Reichler, $\odot$ S. I. Murphy, $\odot$ N. H. Martin, $\odot$ and M. Wiedmann* $\odot$ \\ Milk Quality Improvement Program, Department of Food Science, Cornell University, Ithaca, NY 14853
}

\begin{abstract}
Pseudomonas spp. are important spoilage bacteria that negatively affect the quality of refrigerated fluid milk and uncultured cheese by generating unwanted odors, flavors, and pigments. They are frequently found in dairy plant environments and enter dairy products predominantly as postpasteurization contaminants. Current subtyping and characterization methods for dairy-associated Pseudomonas are often labor-intensive and expensive or provide limited and possibly unreliable classification information (e.g., to the species level). Our goal was to identify a single-copy gene that could be analyzed in dairy spoilage-associated Pseudomonas for preliminary species-level identification, subtyping, and phenotype prediction. We tested 7 genes previously targeted in a Pseudomonas fluorescens multilocus sequence typing scheme for their individual suitability in this application using a set of 113 Pseudomonas spp. isolates representing the diversity of typical pasteurized milk contamination. For each of the 7 candidate genes, we determined the success rate of PCR and sequencing for these 113 isolates as well as the level of discrimination for species identification and subtyping that the sequence data provided. Using these metrics, we selected a single gene, isoleucyl tRNA synthetase $(i l e S)$, which had the most suitable traits for simple and affordable single-gene Pseudomonas characterization. This was based on the number of isolates successfully sequenced for ileS $(113 / 113)$, the number of unique allelic types assigned (83, compared with 50 for 16S rDNA), nucleotide and sequence diversity measures (e.g., number of unique SNP and Simpson index), and tests for genetic recombination. The discriminatory ability of ileS sequencing was confirmed by separation of 99 additional dairy Pseudomonas spp. isolates, which were indistinguishable by $16 \mathrm{~S}$ rDNA sequencing, into 28 different ileS allelic types. Further, we used wholegenome sequencing data to demonstrate the similarities
\end{abstract}

Received July 13, 2020.

Accepted October 25, 2020

*Corresponding author: mw16@cornell.edu in ileS-based phylogenetic clustering to whole-genomebased clustering for 27 closely related dairy-associated Pseudomonas spp. isolates and for 178 Pseudomonas type strains. We also found that dairy-associated Pseudomonas within an ileS cluster typically shared the same proteolytic and lipolytic activities. Use of ileS sequencing provides a promising strategy for affordable initial characterization of Pseudomonas isolates, which will help the dairy industry identify, characterize, and track Pseudomonas in their facilities and products.

Key words: Pseudomonas, subtyping, identification, postpasteurization contamination

\section{INTRODUCTION}

Pseudomonas are ubiquitous in natural environments, nutritionally versatile, often psychrotolerant, and capable of producing undesirable odors, flavors, and pigments that render affected food spoiled and possibly inedible (Hayes et al., 2002; Remenant et al., 2015). For these reasons, Pseudomonas has long been identified as a predominant spoiler of many fresh foods, including uncultured dairy products such as fluid milk and mozzarella cheese (del Olmo et al., 2018; Reichler et al., 2019). Although Pseudomonas spp. are often abundant in raw milk (Rodrigues et al., 2017), heat treatments, such as pasteurization and sterilization, are very effective at reducing their viable populations to well below detectable limits (Macaulay et al., 1963; Dabbah et al., 1971; Weckbach and Langlois, 1977). Because of their sensitivity to heat, the majority of Pseudomonas ultimately responsible for product spoilage are believed to enter dairy products as contaminants following heat treatment, often called postpasteurization contamination (PPC; Schröder, 1984). Poorly cleaned and sanitized dairy processing equipment is often cited as the source of PPC (Griffiths et al., 1984), though PPC may also result from contaminated water aerosols or contamination by worker contact (Sunga et al., 1970; Eneroth et al., 2000).

Researchers have used several subtyping methods to determine the sources of PPC-associated Pseudomonas and to examine product contamination patterns over 
time. Separation-based techniques, such as automatic ribotyping, pulsed-field gel electrophoresis, and randomly amplified polymorphic DNA, have all been used for this purpose (Ralyea et al., 1998; Carrascosa et al., 2015; Carminati et al., 2019). Multilocus sequence typing (MLST), in which several genes are amplified and sequenced to achieve subtyping, has seen broad application for food-associated pathogens such as Listeria spp. and Salmonella (Stessl et al., 2014; Alikhan et al., 2018) and was recently used for subtyping of Pseudomonas associated with color defects in dairy foods (Andreani et al., 2015b). Subtyping based on the DNA sequence of a single housekeeping gene is a less resource-intensive alternative to MLST and has been applied to several groups of organisms, including Listeria spp. and dairyassociated spore-forming bacteria (Durak et al., 2006; Nightingale et al., 2007). Sequencing of $16 \mathrm{~S}$ rDNA has also been used to identify and subtype Pseudomonas and other gram-negative spoilage bacteria in milk, but there are clear limitations to its abilities due to the highly conserved nature of this gene (Masiello et al., 2016; Reichler et al., 2018). Our goal in this study was to identify a single DNA sequencing target in dairy spoilage-associated Pseudomonas that would provide sufficient resolution for species identification as well as initial characterization and subtyping. Utilizing a subtyping technique based on this single target will provide dairy processors with a simple and cost-effective tool for preliminary identification and tracking of Pseudomonas contamination in their facilities and finished products.

\section{MATERIALS AND METHODS}

\section{Pseudomonas Isolate Selection}

All Pseudomonas used in this study were isolated either from pasteurized, homogenized milk, which was incubated at $6^{\circ} \mathrm{C}$ and tested throughout shelf life, or from dairy processing equipment as detailed in the original studies that reported these isolates (Reichler et al., 2018, 2020). Briefly, the milk samples from which the Pseudomonas isolates were derived were collected on multiple dates between July 2015 and January 2018 from 9 different fluid milk plants in the northeast United States. Pseudomonas were isolated both from standard methods agar and from crystal violet tetrazolium agar onto which samples of milk collected over shelf life at $6^{\circ} \mathrm{C}$ were plated. Standard methods agar was incubated at $32^{\circ} \mathrm{C}$ for $48 \mathrm{~h}$, and crystal violet tetrazolium agar was incubated for $48 \mathrm{~h}$ at $21^{\circ} \mathrm{C}$. From both media, morphologically distinct colonies were isolated and identified by partial sequencing of their $16 \mathrm{~S}$ rDNA. A total of 1,297 isolates from these 2 studies were previously confirmed to be Pseudomonas by $16 \mathrm{~S}$
rDNA sequencing and were further characterized by SNP-based subtyping based on an approximately 532bp fragment of the $16 \mathrm{~S}$ rDNA (16S rDNA sequence typing). The isolates were maintained at $-80^{\circ} \mathrm{C}$ in brain heart infusion (BHI) broth (BD, Sparks, MD) with $15 \%$ glycerol. To recover isolates for experimentation, a loopful of each frozen stock was struck onto a plate of BHI agar (BD), and these plates were incubated at $32^{\circ} \mathrm{C}$ for $48 \mathrm{~h}$. Following incubation, an isolated colony of each isolate was inoculated into a test tube containing $5 \mathrm{~mL}$ of BHI broth, and the tubes were incubated at $32^{\circ} \mathrm{C}$ for 18 to $24 \mathrm{~h}$.

To assess the ability of each of the 7 sets of primers to amplify its target gene in a broad diversity of Pseudomonas, a set of Pseudomonas isolates representative of the diversity in the collection was assembled. A total of 113 isolates were selected from the 1,297 available Pseudomonas isolates previously characterized by $16 \mathrm{~S}$ rDNA sequencing (Table 1). These 113 isolates represented 50 different $16 \mathrm{~S}$ sequence types (ST) and originated from a total of 82 milk samples collected from 9 processing facilities on 28 separate collection dates. Isolates were selected to include (1) isolates obtained from multiple processing plants for the 18 most commonly isolated 16S ST (facilitating assessment of discriminatory power) and (2) isolates representing 47 less common $16 \mathrm{~S}$ ST as well as isolates that are genetically distant from the majority of the isolates based on their $16 \mathrm{~S} \mathrm{rDNA}$ sequences (facilitating assessment of inclusivity).

\section{$P C R$ and DNA Sequencing for 7 Candidate Genes Included in a Previously Described MLST Scheme}

Enzymatic lysis was performed on the Pseudomonas isolates to prepare them for PCR. For each isolate, 250 $\mu \mathrm{L}$ of $24-\mathrm{h}$ BHI broth culture (incubated at $32^{\circ} \mathrm{C}$ ) was pelleted by centrifugation $\left(3,220 \times g\right.$ for $10 \mathrm{~min}$ at $\left.4^{\circ} \mathrm{C}\right)$ in a 96-well PCR plate; the pellet was then resuspended in $95 \mu \mathrm{L}$ of $1 \times$ PCR buffer (Promega, Fitchburg, WI). Lysozyme (Thermo Fisher, Waltham, MA) was added to a final concentration of $3 \mathrm{mg} / \mathrm{mL}$, and the solutions were incubated at room temperature for $15 \mathrm{~min}$. Proteinase K (Roche Diagnostics, Mannheim, Germany) was subsequently added to a final concentration of 0.4 $\mathrm{mg} / \mathrm{mL}$, and the solutions were incubated for $1 \mathrm{~h}$ at $58^{\circ} \mathrm{C}$ and then $10 \mathrm{~min}$ at $95^{\circ} \mathrm{C}$ in in an $\mathrm{ABI} 2720$ thermal cycler (Thermo Fisher). The resulting lysates were refrigerated at $4^{\circ} \mathrm{C}$ or frozen at $-20^{\circ} \mathrm{C}$ until the time of use.

Polymerase chain reaction was performed for the 7 genes ( $g \ln S$, gyrB, ileS, nuoD, recA, rpoB, and rpoD) included in a published Pseudomonas fluorescens MLST scheme (Andreani et al., 2015b). The primers, reagents, and reaction conditions used were those described by 
Andreani et al. (2015b). Briefly, a $20-\mu \mathrm{L}$ reaction was prepared for each isolate and each of the 7 target genes (Supplemental Table S1, https://doi.org/10.7298/ rekh-4w25; Reichler et al., 2020b). All reactions then underwent 35 cycles of PCR. The PCR success and amplicon quality were assessed by electrophoresis on a $1.5 \%$ agarose-Tris-borate-EDTA gel, followed by staining with $5 \mu \mathrm{g} / \mathrm{mL}$ ethidium bromide (Amresco, Solon, $\mathrm{OH}$ ) and imaging using a UV transilluminator (GelDoc XR+; Bio-Rad, Hercules, CA). Exonuclease I (10 U) and shrimp alkaline phosphatase $(1 \mathrm{U}$; both from Thermo Fisher) were added to the PCR product, which was incubated at $37^{\circ} \mathrm{C}$ for $45 \mathrm{~min}$ and then at $80^{\circ} \mathrm{C}$ for 15 min to digest the remaining primers and dNTP (Werle et al., 1994). The enzyme-treated PCR products, the appropriate forward or reverse primers, and BigDye 3.1 cycle sequencing chemistry (Thermo Fisher) were used for dye terminator sequencing reactions performed per manufacturer recommendations but with the addition of $4.2 \%$ dimethyl sulfoxide to the reaction mix. Postreaction cleanup and capillary gel electrophoresis were performed by the Biotechnology Resource Center staff (Cornell University, Ithaca, NY) using dye terminator removal columns (Edge BioSystems, Gaithersburg, MD) per manufacturer instructions and an ABI 3730xl capillary DNA analyzer (Thermo Fisher).

\section{Sequence Data Treatment and Allele Assignment}

Sequencher 5.4.5 (Gene Codes Corporation, Ann Arbor, MI) was used to automatically trim the forward and reverse sequence reads based on individual base call quality scores and then to assemble and proofread the sequences obtained from the PCR products. MUSCLE (Edgar, 2004) was used to build a multiple alignment from the proofread sequences for each of the 7 genes, and AliView (Larsson, 2014) was used to trim the aligned sequences to the length used for allelic typing. These trimmed sequences were imported into BioNu- merics 7.6.3 (Applied Maths, Sint-Martens-Latem, Belgium), where the MLST online plugin was used to assign allelic types (AT) to each unique sequence (i.e., differing by $\geq 1$ SNP) for all 7 genes.

\section{Assessment of Primer Specificity and Diversity of Candidate Gene Sequence Products}

Following PCR, sequencing, and generation of proofread and trimmed DNA sequences for each of the 7 genes from the 113 isolates, several diversity metrics were calculated for each candidate gene. DnaSP6 (Rozas et al., 2017) was used to determine the number of nonredundant sequences, the number of polymorphic sites, the total number of mutations, and the nucleotide diversity metric for each gene. The VEGAN package in R (Dixon, 2003; R Core Team, 2019) was used to calculate the Simpson index of diversity from the AT frequency data. RDP4 (Martin et al., 2017) was used to identify putative recombination events for all 7 genes by the RDP, GENECOV, BootScan, MaxChi, Chimaera, SiScan, and 3Seq methods. A putative recombination event was reported when it was detected by 2 or more of these methods.

\section{Assessment of ileS Single-Gene Sequencing- Based Discrimination Compared with 16S rDNA Sequencing-Based Discrimination}

As ileS was identified as the gene most suitable for single-gene sequence typing, the ability of ileS allelic typing to differentiate Pseudomonas was investigated further. For this, the ileS DNA sequences were assessed for 99 isolates sourced from 8 milk processing facilities in the northeast United States between 2015 and 2018. These 99 isolates had indistinguishable $16 \mathrm{~S}$ rDNA sequence typing regions; all of them belonged to $16 \mathrm{~S}$ ST 13 (Reichler et al., 2018, 2020). All isolates characterized here had been collected from finished packaged

Table 1. Distribution of Pseudomonas isolates selected from an initial pool of 1,297 isolates, based on $16 \mathrm{~S}$ rDNA sequence type (ST) data, as a test set for single-gene subtyping

\begin{tabular}{lcccc}
\hline $\begin{array}{l}\text { Frequency of } \\
\begin{array}{l}\text { occurrence of a } \\
\text { given 16S ST }\end{array}\end{array}$ & $\begin{array}{c}\text { No. of 16S ST } \\
\text { within this range }\end{array}$ & $\begin{array}{c}\text { Total no. of isolates } \\
\text { within this range }\end{array}$ & $\begin{array}{c}\text { No. of isolates to } \\
\text { test per 16S ST }\end{array}$ & $\begin{array}{c}\text { Total no. of } \\
\text { isolates to test }\end{array}$ \\
\hline$>0$ and $<5$ & 39 & 89 & $<1^{1}$ & 24 \\
$\geq 5$ and $<10$ & 8 & 45 & 1 & 8 \\
$\geq 10$ and $<50$ & 10 & 223 & 4 & 31 \\
$\geq 50$ and $\leq 100$ & 5 & 364 & $\geq 5^{2}$ & 20 \\
$>100$ & 3 & 576 & - & 113 \\
Total & 65 & 1,297 &
\end{tabular}

${ }^{1} 24$ isolates representing $24 \mathrm{ST}$ were randomly selected for further testing from this group.

${ }^{2} 10,11$, and 9 isolates were selected for the 3 allelic types that fell into this category (ST 9, 13, and 16, respectively). 
milk products or from dairy equipment (rubber gasket) enrichments. The ileS AT assignment for these isolates was performed as described above.

\section{Assessment of ileS Single-Gene Sequencing-Based Discrimination Compared with Whole-Genome Sequencing-Based Discrimination}

Whole-genome sequencing (WGS) was used specifically to investigate a subset of isolates with identical ileS AT that were identified across multiple plants and multiple sample collection dates. The ileS AT that were selected for this part of our study had to meet either of 2 criteria: (1) ileS AT identified in 3 or more samples collected from plant D and in 1 or more samples collected from at least 1 additional plant, or (2) ileS AT identified in 1 or more samples from plant D and in 1 or more samples from each of 2 additional plants. Isolates from the 6 selected ileS AT $(2,5,15,84,89,90)$ all belonged to $16 \mathrm{~S}$ ST 13. A single isolate identified as each selected ileS AT was chosen for WGS from each milk sample in which that AT was detected, for a total of 27 isolates originating from 24 milk samples.

Genomic DNA was extracted and WGS was performed for these 27 isolates using the procedure described by Kovac et al. (2016) with minor modifications. Briefly, purified genomic DNA was extracted from bacterial suspensions using a QIAamp DNA Mini Kit (Qiagen, Valencia, CA), and DNA concentration and purity were checked using a Nanodrop spectrophotometer and a Qubit dsDNA High Sensitivity Kit (both from Thermo Fisher). The DNA samples were submitted the to the Cornell University Institute of Biotechnology Genomics facility in Ithaca, New York, where staff prepared libraries using the Nextera XT DNA Library Preparation Kit (Illumina, San Diego, CA). The libraries were pooled and sequenced in an Illumina HiSeq 2500 rapid run with $2 \times 150$-bp paired-end reads.

The raw sequence reads for all isolates were trimmed using Trimmomatic 0.36 (Bolger et al., 2014), and then FastQC 0.11.5 (https://www.bioinformatics.babraham .ac.uk/projects/fastqc/) was used to check the overall quality of the reads. The reads were screened for contamination using Kraken 2 (https://github.com/ DerrickWood/kraken2) and MetaPhlAn2 (Truong et al., 2015) before they were assembled de novo into contigs using SPAdes 3.13.0 (Bankevich et al., 2012) with the "-careful" pipeline option and a coverage cutoff of 2.0. Contigs shorter than $200 \mathrm{bp}$ were removed, and the quality of the assemblies was checked using QUAST 4.0 (Gurevich et al., 2013). Raw sequence reads and whole-genome sequence assemblies for all sequenced isolates were deposited in NCBI GenBank (https:// www.ncbi.nlm.nih.gov/genbank/) under BioProject
PRJNA579538. Accession ID for individual isolates can be found in Supplemental Table S2 (https://doi.org/ 10.7298/rekh-4w25; Reichler et al., 2020b). Analysis of the sequencing data indicated good sequencing coverage ( 115 to $488 \times$ coverage, mean coverage of $221 \times$ ) and high-quality assemblies (Supplemental Table S2; https: //doi.org/10.7298/rekh-4w25; Reichler et al., 2020b).

To verify the identities of the 27 whole-genome sequenced isolates, their 16S rDNA sequences, extracted using RNAmmer 1.2 (Lagesen et al., 2007), and their ileS sequences, extracted using MLSTcheck (Page et al., 2016), were checked against the sequences previously obtained via PCR and dye-terminator sequencing. To determine species identity, an average nucleotide identity by BLAST (ANIb) distance matrix was constructed using pyani (https://github.com/widdowquinn/pyani). This matrix was constructed using all 27 Pseudomonas whole-genome assemblies along with 8 type strain assemblies downloaded from NCBI GenBank representing the Pseudomonas species P. psychrophila, P. fragi, $P$. deceptionensis, $P$. weihenstephanensis, P. lundensis, P. helleri, P. endophytica, and P. taetrolens. Type strain assemblies were selected for inclusion in the ANIb analysis based on the results of a preliminary neighbor joining tree, constructed in MEGA X (Kumar et al., 2018), using ileS sequences for the 27 newly whole-genome sequenced Pseudomonas isolates as well as 178 Pseudomonas type strains. This phylogeny was used to select the 8 type strain genomes that were most similar to the newly whole-genome sequenced Pseudomonas isolates. The ANIb distance matrix was used to construct a dendrogram in $\mathrm{R}$ using the ggdendro package (de Vries and Ripley, 2016; R Core Team, 2019). For speciation, strains with $>95 \%$ pairwise ANIb were considered to belong to the same species. To compare the phylogenetic relationships and level of resolution between ileSbased and WGS-based discrimination methods, a maximum likelihood tree was constructed based solely on the ileS sequences of the 27 Pseudomonas isolates using RAxML (Stamatakis, 2014), and a maximum likelihood whole-genome SNP tree was constructed using kSNP 3.0 with a k-mer size of 21 for the same 27 isolates (Gardner et al., 2015). To quantitatively compare these 2 phylogenies, the Robinson-Foulds and subtree prune and regraft distance metrics were calculated using the phangorn package in R (Schliep, 2011).

To further assess the ability of ileS allelic typing to discriminate between Pseudomonas spp. and to produce congruent phylogenetic topology for Pseudomonas from a diverse collection, whole-genome assemblies representing 178 Pseudomonas spp. type strains were downloaded from NCBI GenBank. The ileS DNA sequences were extracted from these assemblies using BLAST+ (Camacho et al., 2009) and aligned us- 
ing MUSCLE (Edgar, 2004). The aligned sequences were trimmed to include only the 552-bp region of $i l e S$ used for allelic typing, and this trimmed region was used to construct a maximum likelihood phylogenetic tree with 100 bootstrap replicates using the GTRGAMMA model in RAxML (Stamatakis, 2014). These 178 whole-genome assemblies were also used to construct a bac120 alignment, which consisted of the translated and concatenated partial AA sequences of 120 ubiquitous single-copy proteins, using GTDB-Tk (Parks et al., 2018). This bac120 alignment was used to construct a maximum likelihood phylogenetic tree with 100 bootstraps using the LG Likelihood model in RAxML (Stamatakis, 2014). To compare the phylogeny of this bac120 tree to the tree constructed only from ileS, clusters in both trees were labeled with the Pseudomonas species groups and subgroups delineated by Hesse et al. (2018). In cases where no pre-existing group could be accurately assigned to a given cluster, a new group name was assigned based on the type strain within the cluster that was described the earliest (e.g., a group was assigned the name "P. bauzanensis group" in the ileS tree). The species comprising the assigned groups and subgroups were compared between the bac120 and ileS trees. Deviations in the ileS tree from the group and subgroup assignments in the bac120 tree were determined and used to assess the relative accuracy of ileS-based subtyping for species and group identification. Additionally, the Robinson-Foulds and subtree prune and regraft distance metrics were calculated for the 2 phylogenies using the phangorn package in R (Schliep, 2011).

\section{Isolate Evaluation for Cold-Acting Lipolytic, Proteolytic, and $\beta$-Galactosidic Activity}

A subset of 144 ileS sequenced Pseudomonas isolates from fluid milk were selected to characterize spoilageassociated enzyme activity among the newly generated ileS AT data set. Five isolates were selected from each of the 11 ileS AT that were identified in $\geq 5$ previously ileS sequenced isolates, preferentially choosing isolates from different plants, processing dates, containers, and days of shelf life to yield a total of 55 isolates. One isolate was selected from each of the 89 ileS AT that were identified in $<5$ isolates to yield a total of 89 isolates. Additionally, 4 control isolates were selected: Paenibacillus odorifer FSL H8-0237 (positive) and Viridibacillus arenosi FSL R5-0213 (negative) as $\beta$-galactosidase controls and Pseudomonas sp. FSL R5-0202 (positive) and Raoultella sp. FSL W4-0273 (negative) as controls for both proteolysis and lipolysis. Enzymatic activity was determined at $6^{\circ} \mathrm{C}$ as previously described by Trmčić et al. (2015). Briefly, each selected isolate was inoculated in technical duplicate onto Spirit Blue agar (BD) to assess lipolysis, onto skim milk agar (BD) to assess proteolysis, and onto BHI agar (BD) containing $200 \mathrm{mg} / \mathrm{L}$ X-Gal (Gold Biotechnology, St. Louis, $\mathrm{MO})$ to assess $\beta$-galactosidase activity. All isolates were additionally plated onto BHI agar (BD) for visual assessment of pigment formation. All agar plates were incubated at $6^{\circ} \mathrm{C}$ for $14 \mathrm{~d}$ and then assessed visually for clearing (lipolysis and proteolysis activity) or for blue color appearance ( $\beta$-galactosidase activity).

\section{RESULTS}

\section{ileS Was Identified as the Most Appropriate Target for Single-Gene Sequencing of Diverse Dairy-Associated Pseudomonas}

We performed analyses on the MLST sequence data generated for 113 intentionally selected, dairy-associated Pseudomonas isolates (Table 1) with the goal of selecting the gene with the most favorable combination of metrics for use in single-gene subtyping (Table 2). The number of isolates successfully amplified and sequenced for each gene ranged from a low of 99 (nuoD) to a high of 113 (ileS, rpoB, and rpoD) out of 113 isolates. The number of unique AT (i.e., sequences that differed by $\geq 1$ SNP from any other sequences) ranged from a low of $69(n u o D)$ to a high of $87(g \ln S)$. The number of polymorphic sites and the total number of unique SNP indicate the level of sequence divergence for each gene among the isolates characterized; ileS had the highest values for both the number of polymorphic sites (249) and the total number of unique SNP (384). The average number of pairwise nucleotide differences per site among the sequences for each gene represents another metric for nucleotide diversity, with higher values indicating greater sequence divergence; $\operatorname{gyr} B$ had the highest value at 0.122 . To measure the level of community diversity revealed by each candidate gene, we calculated the Simpson index for each gene using the frequency data for each AT. Simpson index values closer to 1 are indicative of greater community diversity. Although the Simpson index values for all genes were relatively high, $g \ln S$ and recA had the 2 highest values at 0.984 and 0.983, respectively. For comparison with an existing technique, we also examined the $16 \mathrm{~S}$ rDNA sequence metrics for the selected Pseudomonas. Unsurprisingly, each of the 7 candidate genes produced substantially higher values for nonredundant sequences, polymorphic sites, unique SNP, nucleotide diversity, and community diversity than 16S rDNA (Table 2).

Based on these initial findings, we eliminated nuoD (due to low PCR success rate) as well as rpoB and rpoD (both due to low number of unique AT) from 
Table 2. Summary of diversity measures for 7 candidate genes to identify the single gene best suited for Pseudomonas subtyping

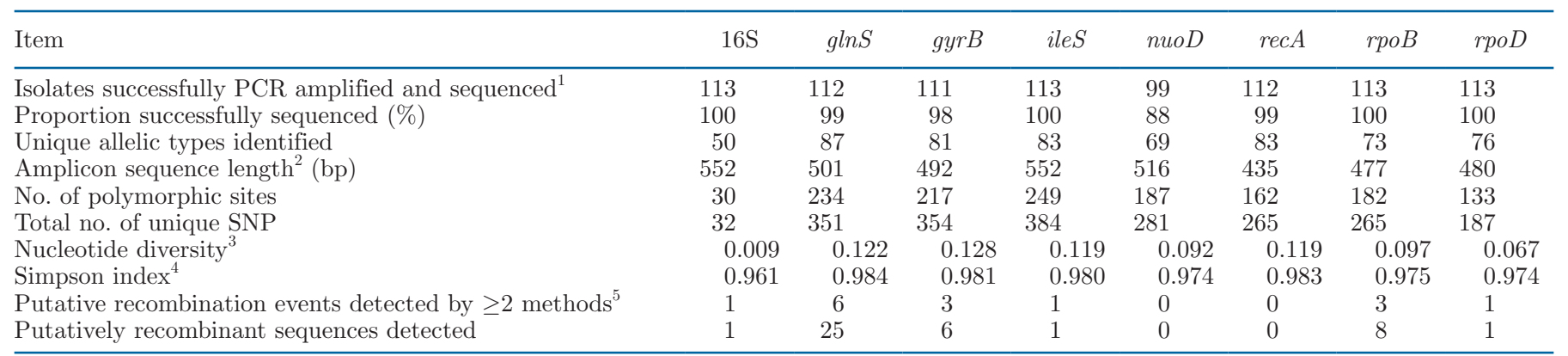

${ }^{1} 113$ representative isolates were selected for PCR and sequencing.

${ }^{2}$ Length of the portion of the PCR amplicon used for analysis.

${ }^{3}$ The average number of nucleotide differences per site between 2 DNA sequences in all possible pairs in the sample population.

${ }^{4} \mathrm{~A}$ statistical measure of diversity that equals 1 - the probability that 2 entities taken at random from the data set represent the same allelic type. Higher values indicate greater diversity.

${ }^{5} \mathrm{RDP} 4$ (Martin et al., 2017) was used to identify putative recombination events for all 7 genes by the RDP, GENECOV, BootScan, MaxChi, Chimaera, SiScan, and 3Seq methods.

consideration. We also analyzed all 7 genes for previous recombination events. Putative recombination events that were detected by $\geq 2$ methods and the number of putatively recombinant sequences are reported in Table 2 . Lower numbers of recombination events were favored in candidate gene selection because they indicate a lower likelihood of phylogenetic relationships being obfuscated due to horizontal gene transfer events. Of the 4 remaining candidate genes, ileS had the second lowest number of putative recombination events $(\mathrm{n}=1)$ and putative recombinant sequences $(\mathrm{n}=1)$. No individual factor was the sole determinant for our selection of a gene target, but we attempted to select the gene with the most favorable combination of metrics. The gene $i l e S$ was selected as it was successfully analyzed for all 113 isolates in the test set; ranked second for nonredundant sequences with 83 , first for both number of polymorphic sites and total number of mutations, third for nucleotide diversity (0.119), and third for Simpson index (0.980); and had only 1 putative recombination event. Successful sequencing for all 113 isolates was important for this decision, as we wanted to ensure that our assay would be broadly applicable across diverse Pseudomonas spp.

\section{ileS Sequencing Allowed for Greater Discrimination of Pseudomonas than 16S rDNA Sequencing}

A collection of 99 Pseudomonas isolates that were all previously characterized as $16 \mathrm{~S}$ rDNA ST 13 were evaluated by ileS allelic typing to compare the discriminatory power of these 2 single-gene sequencing methods. These 99 isolates were differentiated into 28 distinct ileS AT, with between 1 and 10 isolates assigned to each AT. We additionally examined these ileS AT in the context of the milk or environmental samples from which they were obtained. Though all 99 isolates represented the same 16S ST (ST 13), these isolates were collected from a total of 8 plants producing HTST-pasteurized fluid milk (plants A, C, D, G, E, H, J, and I). Notably, 23 ileS AT were identified among isolates obtained from samples collected at plant D over a period of approximately 2 yr (Figure 1). Of these 23 AT, only 6 (AT 1 , $2,86,89,90$, and 92) were ever identified from isolates obtained from samples collected on different processing dates. Repeat isolation of these AT over time suggests, but does not prove, environmental persistence of certain Pseudomonas within plant D. A total of 9 ileS AT (AT 2, 5, 15, 29, 84, 89, 90, 91, and 98) were isolated from multiple plants (Figure 1); for example, AT 15 was isolated from plants D, H, J, and C.

\section{ileS Phylogeny Generally Agreed with WGS-Based Phylogeny}

To evaluate the accuracy of phylogenetic relationships inferred among closely related isolates based on ileS sequence data, we performed WGS on 27 Pseudomonas isolates that had the same 16S ST but represented 6 ileS AT. As revealed by ANIb analysis (Figure 2; Supplemental Table S3, https://doi.org/10.7298/rekh $-4 \mathrm{w} 25$; Reichler et al., 2020b), the sequence diversity ranged from relatively minor (e.g., isolates with ileS AT 2, 89, and 90 each shared $>99.4 \%$ identity within their respective AT) to somewhat greater (e.g., the 5 isolates with ileS AT 15 shared $>97.8 \%$ identity, the 4 isolates with ileS AT 5 shared $>97.6 \%$ identity, and the 5 isolates with ileS AT 84 shared $>96.3 \%$ identity). Using a generally recognized species cutoff of $>95 \%$ identity, the 27 Pseudomonas isolates characterized here by 
WGS represent 3 distinct species, though all 27 isolates were identified as the same ST using $16 \mathrm{~S}$ rDNA-based subtyping. Only 1 of these species could be assigned an existing name based on a cutoff of $>95 \%$ : ileS AT 2,5 , 15, and 89 were identified as Pseudomonas helleri; ileS AT 84 and AT 90 most likely represent 2 separate species for which no type strain whole-genome sequences were publicly available at the time of analysis. No ileS AT were identified as containing more than 1 species. This result further supports that ileS AT assignments show greater resolution and are better aligned with speciation than $16 \mathrm{~S}$ rDNA alone.

We also compared maximum likelihood phylogenetic trees generated from (1) a whole-genome SNP matrix constructed from the 27 Pseudomonas whole genomes and (2) the aligned ileS sequences extracted from the same 27 genomes (Figure 3). Each AT, by definition, represented a monophyletic group within the ileS tree. However, in the SNP tree, AT 90, 84, 2, and 89 remained monophyletic groups, whereas AT 15 and 5 represented paraphyletic groups. For AT 15, all isolates were inferred to have descended from a common ances- tor that was shared with AT 2, AT 5, and AT 89. For AT 5, all isolates were inferred to descend from a common ancestor shared with AT 89. The low bootstrap support for some nodes in the ileS tree is due to the close genetic similarity of the different AT included (as few as $3 \mathrm{SNP}$ in a 552-bp ileS sequence). Despite this, the clustering observed in the whole-genome SNP tree was generally preserved in the ileS tree. The normalized Robinson-Foulds distance between these 2 trees was 0.667 , meaning that these trees differ from each other by $66.7 \%$ of their maximal possible distance. The subtree prune and regraft distance calculated for the 2 trees was 6 , meaning that the topological differences between the trees may be explained by a minimum of 6 subtree prune and regraft operations.

To substantiate that ileS AT-based groupings would also be effective across diverse Pseudomonas, an ileSbased tree for 178 type strain Pseudomonas was compared with a bac120-based tree to assess the ability of ileS to accurately assign phylogenetic relationships (Figure 4; Supplemental Figure S1, https://doi.org/10 .7298/rekh-4w25; Reichler et al., 2020b). In both trees,

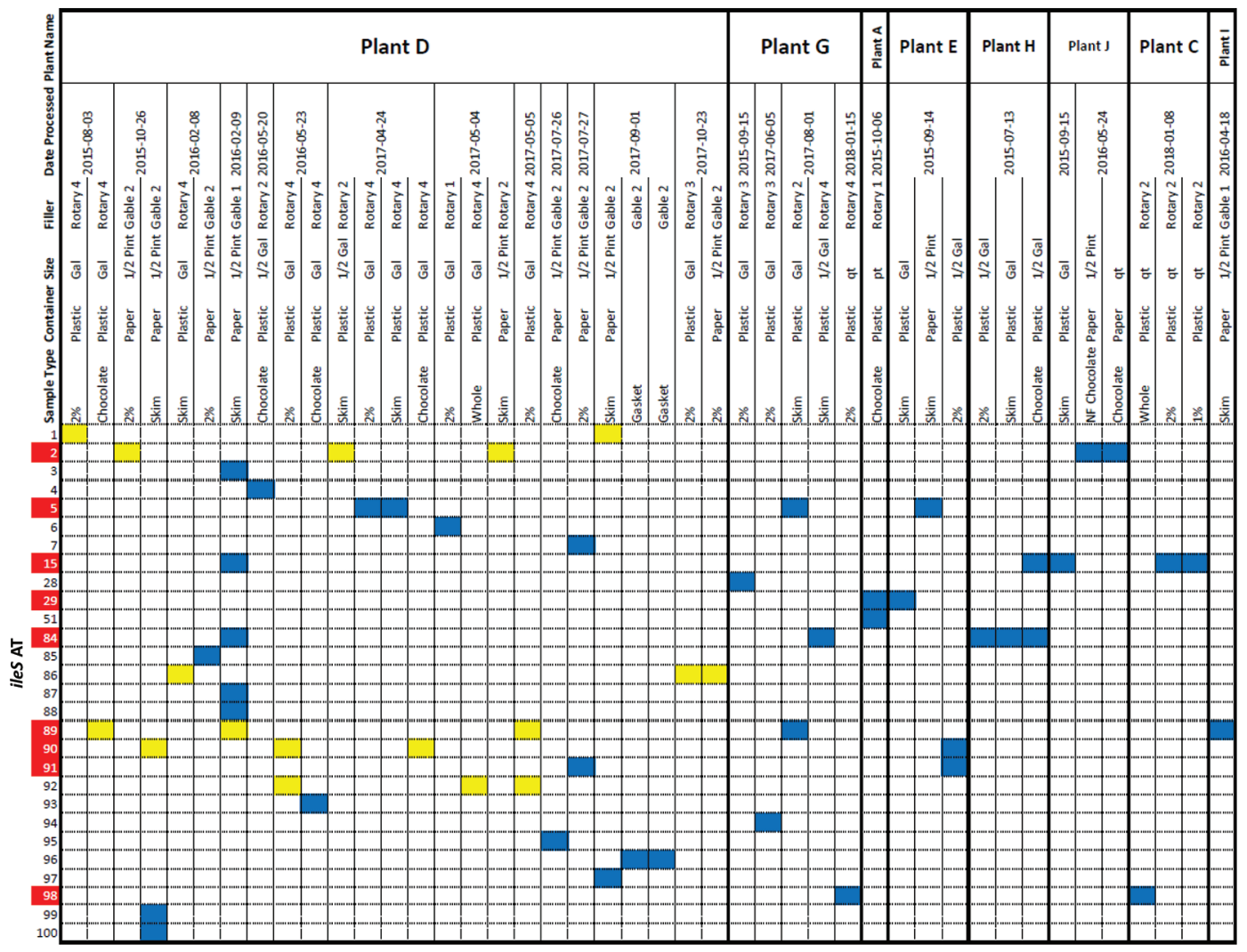

Figure 1. The ileS allelic typing results for isolates with $16 \mathrm{~S}$ rDNA sequence type 13 from multiple dairy plants. Dairy plant designations are consistent with designations in previous studies that reported spoilage patterns in these facilities (Reichler et al., 2018, 2020). Each column represents an individual product or environmental sample, and each row represents an ileS allelic type (AT). Dates (yr-mo-d) when samples were collected are indicated at the top of each column. Red indicates ileS AT found at multiple plants, yellow indicates AT found on multiple sampling dates at 1 plant, and blue indicates AT found on a single sampling date in only 1 given plant. 


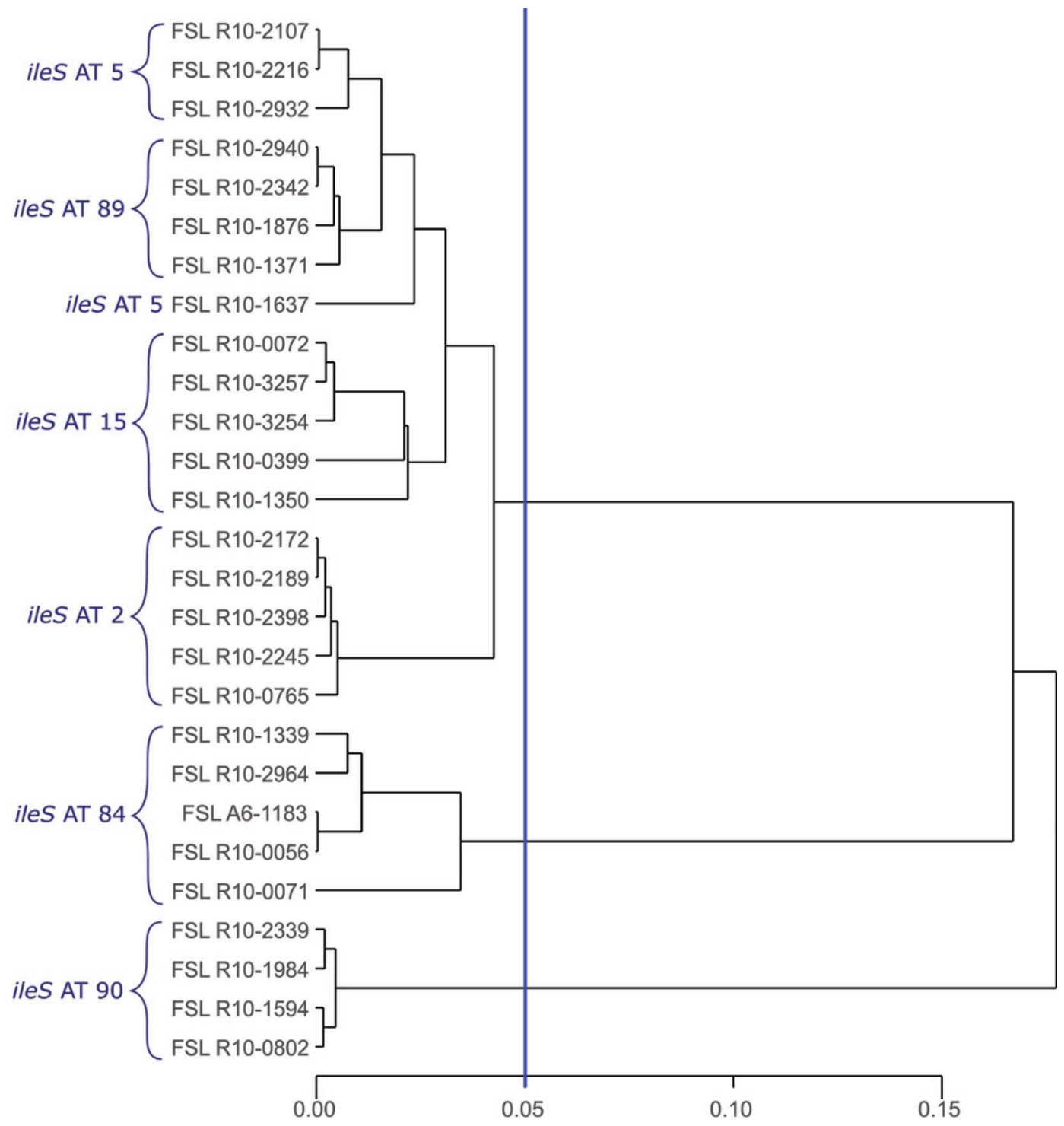

Figure 2. Average nucleotide identity by BLAST (ANIb) dendrogram based on pairwise distances between the whole-genome sequences of the 27 Pseudomonas isolates with $16 \mathrm{~S}$ sequence type 13. The scale bar represents pairwise distance, and the vertical line represents a pairwise distance of 0.05 (i.e., $95 \%$ ANIb), the typical cutoff for speciation. AT = allelic type. Isolates are identified by their Food Safety Laboratory (FSL) collection names.

bootstrap support for many nodes outside of the $P$. fluorescens group was generally low $(<40 \%)$. The ileS sequence data alone were able to distinguish 176 of the 178 type strains ( $P$. meliae and $P$. savastanoi being indistinguishable), whereas bac120 was able to distinguish between all 178 type strains. For the clades in the bac120 tree that were strongly supported by bootstrap values $(>90 \%)$, the ileS-based phylogeny generally provided similar clustering of both Pseudomonas groups and subgroups. For example, the same set of strains was classified into the $P$. putida group, supported by bootstrap values of $100 \%$ and $74 \%$ for bac 120 and ileS, respectively. Within the $P$. fluorescens group (which includes 9 subgroups), 51 of 66 type strains were placed into the same subgroup in both the ileS and bac120 trees (Figure 4); most notably, in the ileS phylogeny, some type strains were placed in other subgroups $(P$. gessardii and $P$. koreensis) or did not form clusters $(P$. mandelii). Outside of the $P$. fluorescens group, 94 of the 112 remaining type strains were placed into the same group in both the ileS and bac120 trees (Figure 4; Supplemental Figure S1, https://doi.org/10.7298/ rekh-4w25; Reichler et al., 2020b). Notable here was the loss of monophyly for the $P$. anguilliseptica and $P$. pertucinogena groups in the ileS phylogeny, which separated each of these groups into 2 clades. The nor- 
malized Robinson-Foulds distance between the ileS and bac120 trees was 0.650 , whereas the subtree prune and regraft distance calculated for the 2 trees was 59. However, these tree distance metrics are typically used for comparison between different methods of phylogenetic reconstruction rather than comparison between phylogenies built from different data sets and should thus be interpreted with caution. Overall, for a broad diversity of Pseudomonas isolates, the ileS phylogeny provided classification into species groups and subgroups that are largely consistent with bac120 classification

\section{Pseudomonas Spoilage-Relevant Enzyme Production Was Associated with ileS Clade}

A total of 144 representative Pseudomonas isolates from pasteurized milk samples were used to assess production of spoilage-associated enzymes and association of these phenotypes with ileS AT (Figure 5). The majority of the evaluated isolates $(n=126)$ belonged to various subgroups within the $P$. fluorescens group, whereas 18 isolates clustered into the P. putida, P. rhizosphaerae, $P$. anguilliseptica, and $P$. lutea groups. None of these 144 isolates exhibited $\beta$-galactosidase activity. Overall, the ileS phylogeny segregated isolates according to spoilage-associated enzyme production. For instance, isolates within the $P$. fragi and $P$. chlororaphis subgroups of the $P$. fluorescens group were generally lipolytic (56/69 and 2/2, respectively) and nonproteolytic ( $67 / 69$ and $2 / 2$, respectively), whereas the remaining subgroups within the $P$. fluorescens group present in the data set $(P$. koreensis and $P$. fluorescens 1 and 2 ) were both lipolytic (5/5 and $32 / 32$, respectively) and proteolytic (5/5 and $29 / 32$, respectively). The isolates within the $P$. putida group were generally lipolytic $(6 / 8)$ and nonproteolytic (8/8). Overall, production of spoilage-associated enzymes was generally delineated by Pseudomonas species groups and subgroups as determined by ileS. Isolates that produced yellow pigment on BHI agar were generally restricted to the $P$. rhizosphaerae group (5/5 within the group, 2/139 outside the group; Figure 5).

\section{Whole-Genome SNP iles}

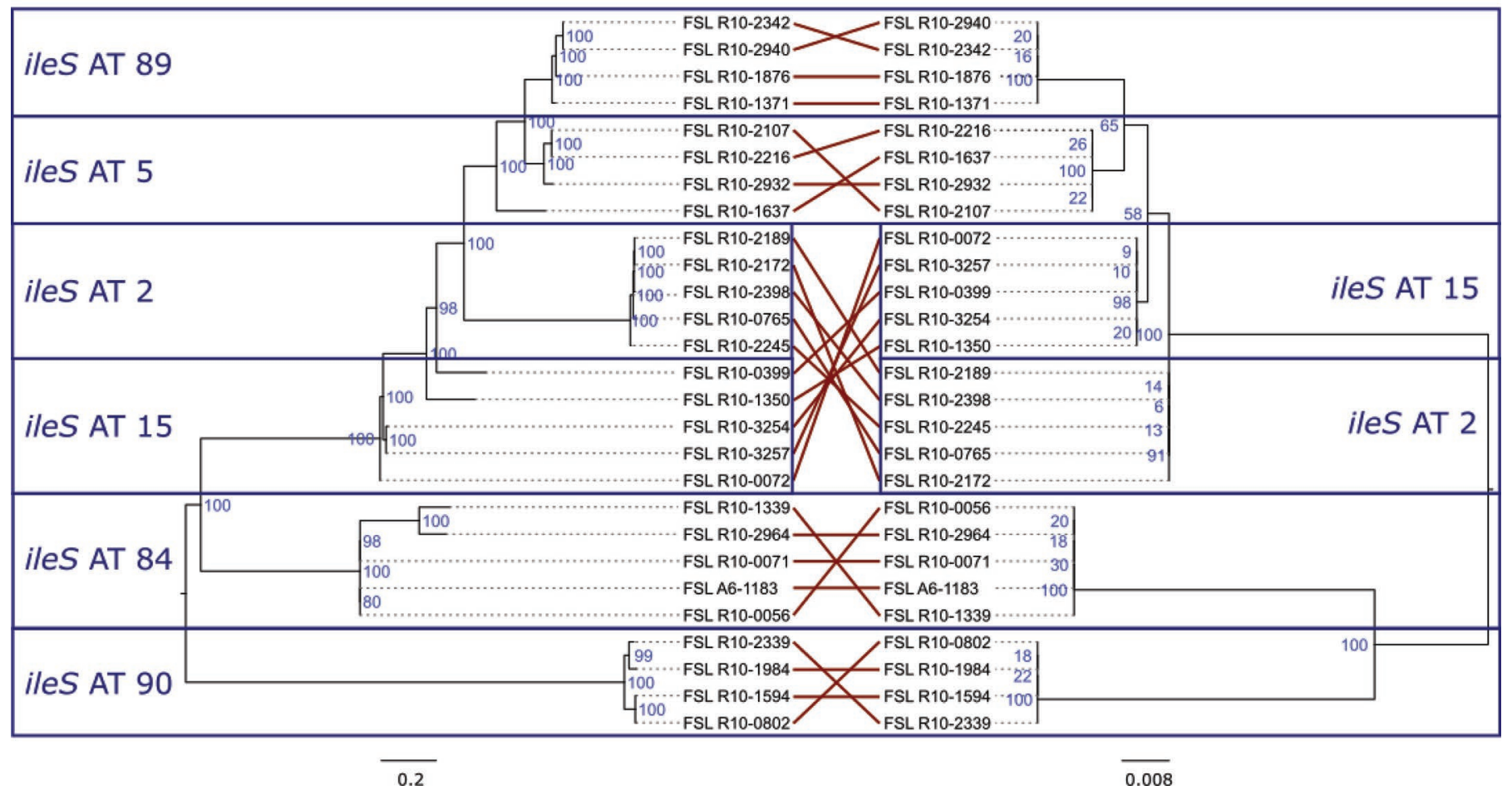

Figure 3. Maximum likelihood phylogenies of 27 newly sequenced Pseudomonas isolates, generated from a whole-genome SNP matrix (left) and the 552-bp ileS allelic typing sequences (right). All 27 isolates represented the same $16 \mathrm{~S}$ rDNA sequence type (sequence type 13); the same whole-genome sequencing data were used to generate the average nucleotide identity BLAST dendrogram shown in Figure 2. Both trees are drawn to scale and midpoint rooted. In the whole-genome SNP tree, the scale bar represents 0.2 nucleotide substitution per site. In the $i l e S$ tree, the scale bar represents 0.008 nucleotide substitution per site. Numbers in blue located next to the nodes represent node confidence as the percentage of 100 bootstrap replicates. Red lines are drawn to join identical tips in the 2 trees. Blue boxes circumscribe individual ileS allelic types (AT). Isolates are identified by their Food Safety Laboratory (FSL) collection names. 


\section{DISCUSSION}

Although Pseudomonas is a major dairy spoilage organism and is relevant and important across a range of perishable dairy products, simple and affordable tools for Pseudomonas identification (e.g., to the level of Pseudomonas species group, subgroup, or species) and initial characterization (e.g., subtype and spoilage phenotype) are still largely undescribed. Thus, we developed and initially assessed a single-gene sequencingbased approach (targeting ileS) to identify and characterize dairy-associated Pseudomonas isolates. With the initial isolate set evaluated here, we have shown that (1) ileS sequencing allows for preliminary species prediction and initial subtype discrimination for a broad range of dairy-associated Pseudomonas isolates and (2) specific ileS AT are associated with the production of selected spoilage enzymes (i.e., lipase and protease) at $6^{\circ} \mathrm{C}$. Hence, ileS sequencing represents a promising tool for the dairy industry.

\section{iles Sequencing Allows for Pseudomonas Isolate Species Prediction and Initial Subtype Discrimination}

Several identification and subtyping methods have been described for Pseudomonas; however, many of them focus on specific groups or species (e.g., P. syringae, P. aeruginosa; Sarkar and Guttman, 2004; Johnson et al., 2007; van Belkum et al., 2015). In addition, many characterization methods previously described are costly, time consuming, or require specialized expertise and equipment to complete (e.g., pulsed field gel electrophoresis, randomly amplified polymorphic DNA,

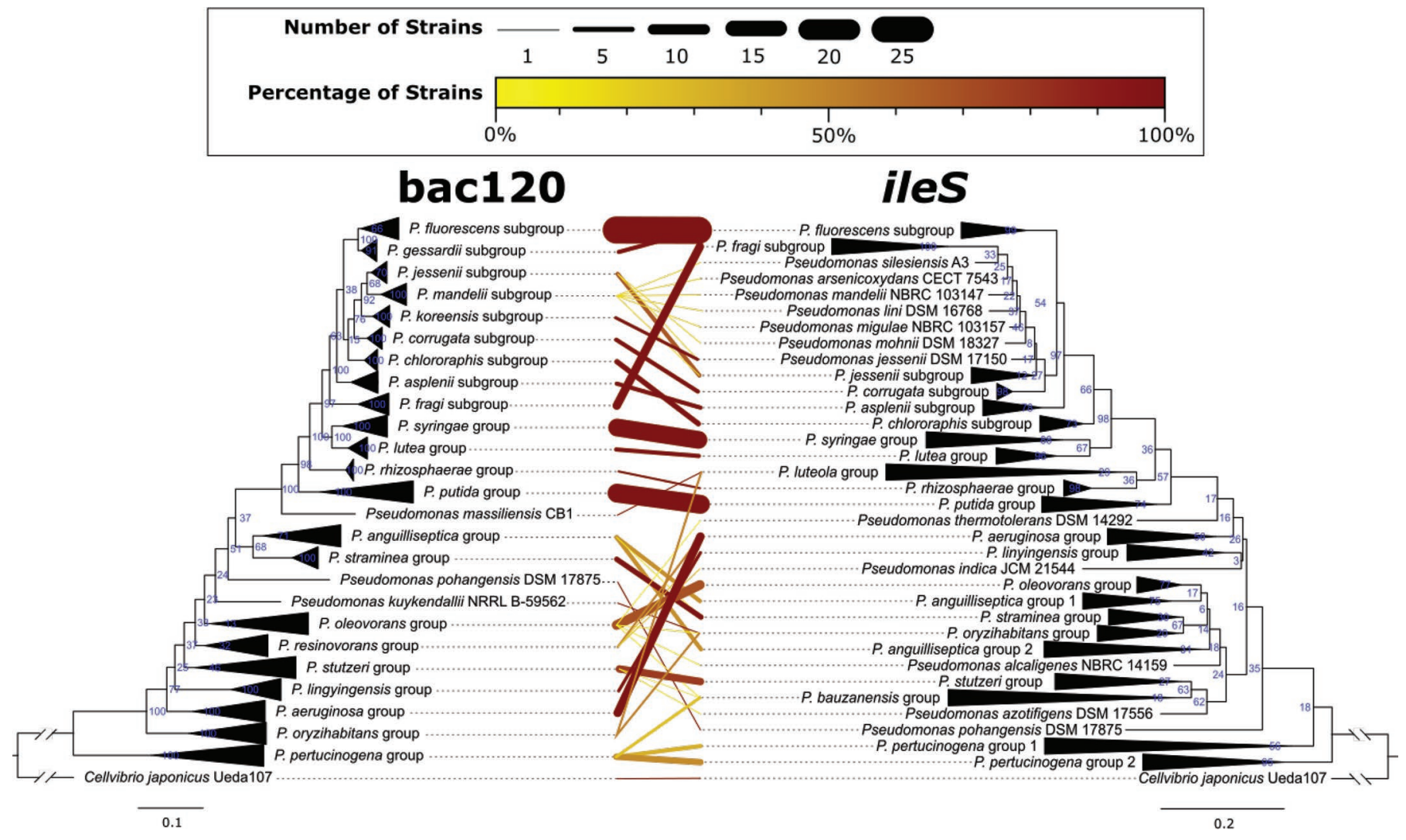

Figure 4. Maximum likelihood phylogenetic trees of 178 Pseudomonas spp. type strains based on concatenated partial AA sequences of 120 single-copy housekeeping genes (bac120, left) and on the 552-bp ileS allelic typing sequence (right). Whole-genome sequences for these type strains were downloaded from NCBI GenBank, and ileS sequences were extracted from the respective assemblies. Cellvibrio japonicus Ueda 107 was used as an outgroup to root the trees, and both trees are drawn to scale, with the exception of this truncated outgroup branch. In the bac120 tree, the scale bar represents 0.1 AA substitution per site. In the ileS tree, the scale bar represents 0.2 nucleotide substitution per site. Numbers in blue located next to the nodes represent node confidence as the percentage of 100 bootstrap replicates. The black triangles located on terminal branches represent condensed monophyletic clusters containing 2 or more type strains. The lines drawn between the tip labels of the 2 trees connect tips containing $\geq 1$ of the same type strains. The line width represents the number of type strains shared between the 2 tips, and the line color represents $100 \times$ (no. of type strains represented by the line)/(no. of type strains represented by the bac120 tree tip) - that is, the percentage of the total number of type strains originating from a given tip in the bac120 tree included in the line originating from that tip. Uncondensed versions of these trees are included as Supplemental Figure S1 (https://doi.org/10.7298/rekh-4w25; Reichler et al., 2020b). 


\begin{tabular}{|l|}
\hline Phenotype $\quad+$ \\
\hline Proteolysis \\
\hline Lipolysis \\
\hline Yellow Pigment \\
\hline
\end{tabular}

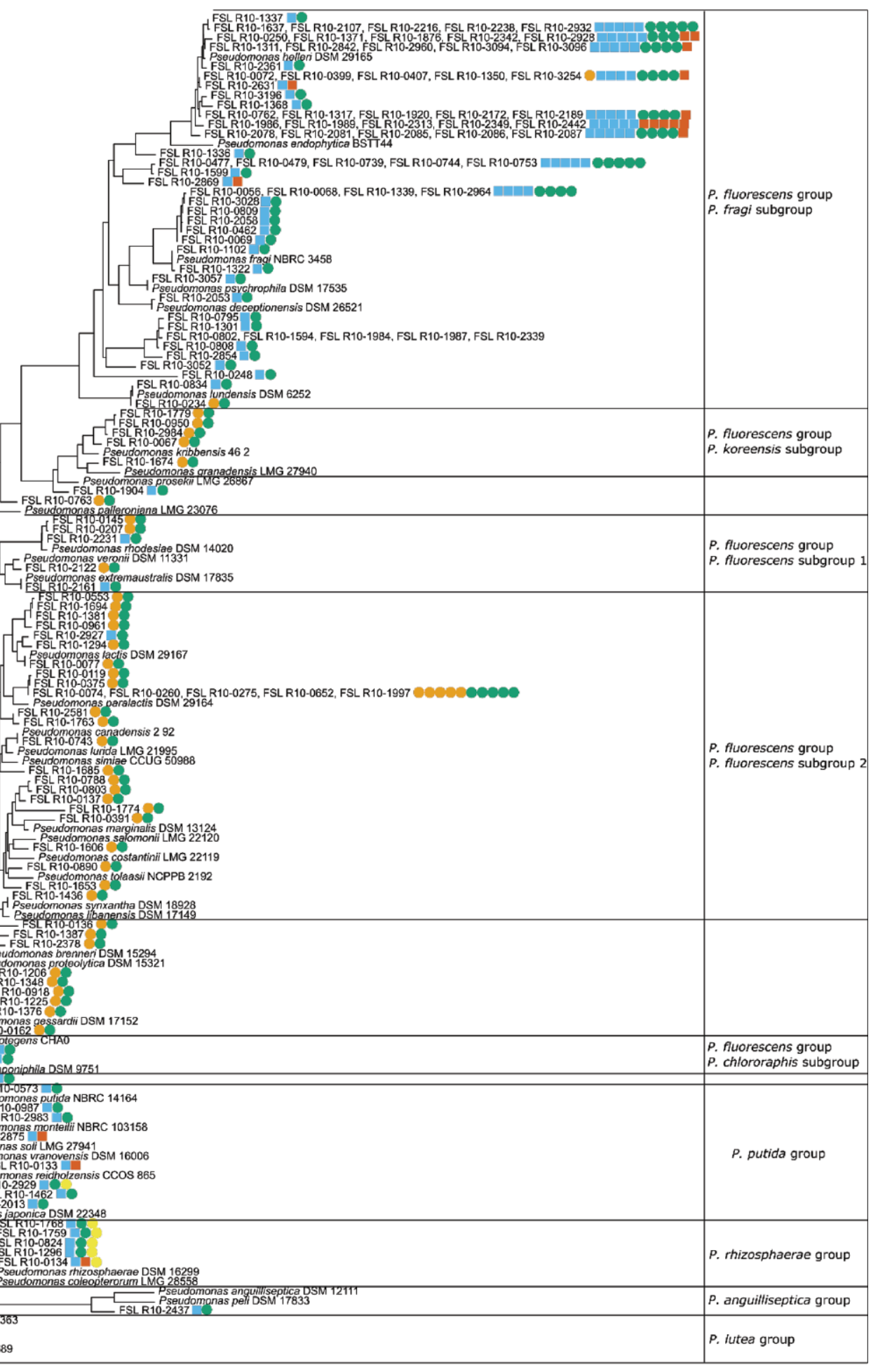

Figure 5. Maximum likelihood phylogeny of Pseudomonas spp. based on a multiple alignment of sequenced ileS alleles. Branch lengths correspond to the average number of mutations per site. The scale bar represents 0.1 nucleotide substitution per site. Colored shapes represent spoilage-associated enzyme production for each given isolate or isolates (determined at $6^{\circ} \mathrm{C}$ ) or production of yellow pigments. For spoilageassociated enzyme production, a circle represents detection of enzymatic activity $(+)$, and a square represents absence of detectable enzymatic activity $(-)$. For yellow pigment production, only isolates that were positive for the pigment production are marked with a yellow circle. Branch labels prefixed with "FSL" identify isolates that were used here for ileS sequencing and phenotypic characterization; branch labels listing genus and species are type strains, and no phenotypic characterization was performed for these isolates. 
WGS, CRISPR spacer analysis, and MLST; Eneroth et al., 2000; Porteous et al., 2002; Botes et al., 2003; van Belkum et al., 2015). Although 16S rDNA sequencing provides a universal approach to initial identification of bacterial isolates, its low discriminatory power limits the usefulness of this approach for investigation of contamination and spoilage issues in dairy. As singlegene sequencing-based approaches have shown promise for affordable and rapid initial classification of several other bacterial groups, including bacterial groups associated with dairy spoilage (Durak et al., 2006), we first set out to define a single gene target that could be used with the Pseudomonas typically associated with dairy products. Beginning with 7 possible target genes from a Pseudomonas-specific MLST scheme (Andreani et al., 2015b), we identified ileS as the most suitable target for single-gene speciation and subtyping purposes. Key criteria for selection of ileS included (1) high discriminatory power, (2) low recombination frequency, and (3) high level of inclusivity, defined as ability to yield PCR products and sequence data for a range of Pseudomonas isolates obtained from fluid milk. We elected to begin our search with genes included in a pre-existing MLST scheme (Andreani et al., 2015b) as this (1) took advantage of previous data that demonstrated the suitability of these 7 genes and (2) allowed for backward compatibility with previous subtyping efforts. Our general approach described here could, however, also be used to identify candidate genes stating with larger genes sets (e.g., core genes defined based on WGS data).

Evaluation of ileS discriminatory power against both (1) the other 6 genes included in the previously reported MLST scheme (i.e., glnS, gyrB, nuoD, recA, $r p o B$, and rpoD) and (2) sequencing a fragment of $16 \mathrm{~S}$ rDNA confirmed that the highly conserved nature of the $16 \mathrm{~S}$ rDNA limits its value for species identification and determination of intrageneric and intraspecific relationships (Moore et al., 1996; Anzai et al., 2000; Yamamoto et al., 2000). Several previous studies have assessed multi-gene sequencing-based approaches for classification and phylogenetic characterization of Pseudomonas; however, most have used a combination of at least 2 genes (e.g., gyr $B$ and rpoD; Yamamoto et al., 2000). Previously evaluated genes included $a p r X$, atpD, carA, gyrB, oprF, recA, rpoB, and rpoD (Yamamoto et al., 2000; Bodilis et al., 2004; Hilario et al., 2004; Ait Tayeb et al., 2005; Caldera et al., 2016); gyrB, recA, $r p o B$, and $r p o D$ were also evaluated in our study, albeit using different PCR primers. The gene ileS was found to possess a superior combination of inclusivity and diversity traits compared with each of these 4 genes. An additional challenge with $16 \mathrm{~S}$ rDNA sequencing is that many bacterial genomes, including those of Pseudomonas spp., contain multiple rDNA operons and hence multiple 16S rRNA gene copies (Hartmann et al., 1986; Fogel et al., 1999), which often differ from each other in nucleotide sequence. For instance, Pseudomonas putida KT2440 contains 7 copies of $16 \mathrm{~S}$ rDNA that represent 4 distinct alleles separated by 3 SNP (Acinas et al., 2004). When universal 16S primers are used for PCR amplification, each of these different copies may be amplified, which yields Sanger sequencing data with ambiguous bases that are difficult to analyze. This makes reliable AT assignment based on 16S rDNA sequencing data extremely challenging. By comparison, sequence data for a single-copy gene such as ileS can be analyzed much more easily. Identification of isolates with the same ileS AT from multiple facilities suggests, however, that the discriminatory power of ileS allelic typing sensitivity may in some cases not be sufficient. Confirmation of a close relationship between 2 isolates with identical ileS types may require further characterization (e.g., by WGS), as also supported by our data showing that a single ileS AT can contain isolates that are found to be distinct by WGS. Nonetheless, our data showed that ileS generated 28 unique AT from 99 isolates, whereas $16 \mathrm{~S}$ rDNA sequence typing identified only $1 \mathrm{ST}$, demonstrating substantial improvement in subtyping sensitivity.

Our results also suggest that ile $S$ sequences can provide appropriate data for initial clustering of isolates into groups that can be correlated with Pseudomonas species and Pseudomonas species groups and subgroups. Although limited evidence of recombination among Pseudomonas ileS sequences provided initial indication that recombination is unlikely to frequently obscure the phylogenetic relationship of isolates, singlegene sequencing is not expected to allow for reliable reconstruction of phylogenetic relationships, as also reported for several other organisms, including Listeria spp. (Liao et al., 2017) and gram-positive aerobic sporeforming bacteria (Durak et al., 2006). The limitation of ileS-based phylogenies is further illustrated by the fact that the utilized portion of ileS is 552 bp in length, whereas other methods use substantially more information to infer phylogenies. For instance, the SNP-based tree in Figure 3 relied on a matrix of $>56,000 \mathrm{SNP}$, and the bac120 tree in Figure 4 was derived from a concatenated series of 5,035 AA residues. Even with these limitations, our comparison of an ileS phylogeny with a bac120 phylogeny for the 178 Pseudomonas type strain isolates with available WGS data showed similar overall clustering. In general, clustering provided sufficient information to allow for species group assignment based on ileS data, even though ileS phylogeny in several cases did not infer the correct evolutionary relationship of isolates (assuming that the bac120 phylogeny represents the true evolutionary relationships 
of the isolates). Although nucleotide similarity cutoffs have been defined for species assignment based on WGS (e.g., ANIb of $\geq 95 \%$ ), defining cutoffs based on singlegene sequences tends to be extremely challenging, if not impossible, presenting a limitation to the application of ileS for species identification. Hence, we conclude that although ileS data can in most cases be used to correctly assign isolates to taxonomic groups, reliable identification of isolates with ileS sequences that are distinct from type strains may require more sophisticated analysis (e.g., WGS) to allow for initial taxonomic group or species assignment. For instance, Hesse et al. (2018) produced a phylogeny based on the protein sequences of 100 single-copy orthologous genes derived from the whole-genome sequences of 163 Pseudomonas species. A more generalized approach to a similar end result is described by Parks et al. (2018), whose bac120 phylogeny was used in this study. Though these contemporary methods offer unparalleled resolution and accuracy in phylogenetic placement of species, they impose several hurdles that prevent broad adoption by dairy processors at this time. Hence, routine use of ileS sequencing (compared with routine WGS) is likely to be valuable. Dairy plants and their service laboratories could use this approach to diagnose, identify, and track PPC in their facilities. Although routine use of ileS may be limited in the short to medium term, these tools are likely to be viable in cases of major spoilage incidents, as supported by the use of more costly techniques to investigate high-profile spoilage cases in the past (e.g., pulsed-field gel electrophoresis or WGS; Martin et al., 2011; Andreani et al., 2015a).

\section{ileS AT Is Associated with Relevant Spoilage Enzyme Production}

A subset of the isolates that were characterized here by $i l e S$ sequencing were screened for the expression of relevant spoilage enzymes on laboratory media. These data revealed differences in proteolysis and lipolysis expression between Pseudomonas species groups and subgroups, namely that the majority of $P$. putida group and $P$. fragi subgroup isolates are lipolytic and nonproteolytic, whereas the majority of $P$. fluorescens and $P$. koreensis subgroup isolates are both lipolytic and proteolytic. Similar enzymatic evaluations of dairyassociated Pseudomonas were performed by Wiedmann et al. (2000) and Dogan and Boor (2003), both of whom demonstrated that unique clusters of Pseudomonas spp. exhibited different enzyme activities. Specifically, both publications reported that clusters consisting of $P$. fluorescens often exhibit both proteolytic and lipolytic activity, which agrees with our findings, but also that isolates identified as $P$. fragi and $P$. putida generally lack both proteolytic and lipolytic activity, which differs from our findings. Factors that may account for these discrepancies include the use of differently formulated media for enzymatic evaluation, enzyme evaluation at $30^{\circ} \mathrm{C}$ in these previous studies rather than $6^{\circ} \mathrm{C}$ (as performed here), and the use of phenotypic characterization rather than DNA sequencing for species assignment. More recent studies (Meng et al., 2017, 2018), however, included classification of dairy-associated Pseudomonas spp. by DNA sequencing (producing more reliable species assignments) as well as evaluation of isolates for enzymatic activity. For example, a study reported that Pseudomonas spp. isolated from raw goat, camel, buffalo, and yak milk generally exhibited low temperature proteolysis for the species P. fluorescens, P. putida, and P. fragi, similar to our findings reported here; isolates in this study were identified by $16 \mathrm{~S}$ rDNA and $r p o B$ sequencing (Meng et al., 2018). In contrast, Pseudomonas spp. isolated from raw bovine milk exhibited much more variation than we observed in low-temperature proteolysis for these 3 species groups (Meng et al., 2017). Identification of spoilage-associated enzymatic activities in pure culture systems provides initial data on spoilage capabilities of isolates; however, it should be noted that spoilage expression in fluid milk may depend on additional variables, such as prior heat treatment, storage conditions, and presence of additional types of bacteria (Worm et al., 2000; Nicodème et al., 2005; Colantuono et al., 2020). Future studies may benefit from correlating spoilage phenotypes with presence and genotype of the $\operatorname{apr} X$-lip $A$ operon, which encodes extracellular protease and lipase enzymes that may be associated with product degradation (Woods et al., 2001; Zhang et al., 2019). Spoilage enzyme association with specific ileS AT has several potential applications in dairy plant management, including assistance in root cause analysis following spoilage incidents, evaluation of long-term trends in product quality, and prioritization of strains that produce spoilage enzymes for corrective actions related to $\mathrm{PPC}$.

\section{CONCLUSIONS}

Although WGS of bacterial isolates and the interpretation of the resulting data are still beyond the capabilities of most dairy processors, Pseudomonas subtyping based on the DNA sequence of ileS can provide practical and actionable information to dairy processors. Data from ileS sequencing are easily interpreted, provide greater resolution for Pseudomonas than partial $16 \mathrm{~S}$ rDNA sequencing, and are useful for species identification, initial identification of possible persistent contamination issues, and prediction of enzy- 
matic spoilage potential. With the disadvantages of $16 \mathrm{~S}$ rDNA sequencing, ileS sequencing for Pseudomonas could be a cost-effective, resource-minimal, and yet still informative tool. Single-gene sequencing is presently far more affordable than WGS and can therefore be used both for larger numbers of isolates and by processors with fewer resources. Application of tools such as ileS subtyping may thus be valuable to dairy processors working toward improved product quality and shelf life. Furthermore, single-gene targets such as ileS or rpoB (described previously as facilitating discrimination of Bacillus) could be applied in targeted metagenomics assays designed to characterize spoilage organisms.

\section{ACKNOWLEDGMENTS}

This work was supported by a grant from bioMérieux (Hazelwood, MO; OSP \# 83959) and by the continued financial support of the New York State Dairy Promotion Advisory Board (Albany, NY; OSP \# 83562) through the New York State Department of Agriculture and Markets (Albany, NY). Martin Wiedmann has served as an uncompensated member of the bioMérieux Molecular Advisory Group. The remaining authors have not stated any conflicts of interest.

\section{REFERENCES}

Acinas, S. G., L. A. Marcelino, V. Klepac-Ceraj, and M. F. Polz. 2004. Divergence and redundancy of $16 \mathrm{~S}$ rRNA sequences in genomes with multiple rrn operons. J. Bacteriol. 186:2629-2635. https:// doi.org/10.1128/JB.186.9.2629-2635.2004.

Ait Tayeb, L., E. Ageron, F. Grimont, and P. A. D. Grimont. 2005. Molecular phylogeny of the genus Pseudomonas based on rpoB sequences and application for the identification of isolates. Res. Microbiol. 156:763-773. https://doi.org/10.1016/j.resmic.2005.02 .009 .

Alikhan, N.-F., Z. Zhou, M. J. Sergeant, and M. Achtman. 2018. A genomic overview of the population structure of Salmonella. PLoS Genet. 14:e1007261. https://doi.org/10.1371/journal.pgen .1007261

Andreani, N. A., L. Carraro, M. E. Martino, M. Fondi, L. Fasolato, G. Miotto, M. Magro, F. Vianello, and B. Cardazzo. 2015a. A genomic and transcriptomic approach to investigate the blue pigment phenotype in Pseudomonas fluorescens. Int. J. Food Microbiol. 213:88-98. https://doi.org/10.1016/j.ijfoodmicro.2015.05.024.

Andreani, N. A., M. E. Martino, L. Fasolato, L. Carraro, F. Montemurro, R. Mioni, P. Bordin, and B. Cardazzo. 2015b. Reprint of "Tracking the blue: A MLST approach to characterise the Pseudomonas fluorescens group." Food Microbiol. 45(A):148-158. https:/ /doi.org/10.1016/j.fm.2014.11.011.

Anzai, Y., H. Kim, J. Y. Park, H. Wakabayashi, and H. Oyaizu. 2000. Phylogenetic affiliation of the pseudomonads based on 16S rRNA sequence. Int. J. Syst. Evol. Microbiol. 50:1563-1589. https://doi .org/10.1099/00207713-50-4-1563.

Bankevich, A., S. Nurk, D. Antipov, A. A. Gurevich, M. Dvorkin, A. S. Kulikov, V. M. Lesin, S. I. Nikolenko, S. Pham, A. D. Prjibelski, A. V. Pyshkin, A. V. Sirotkin, N. Vyahhi, G. Tesler, M. A. Alekseyev, and P. A. Pevzner. 2012. SPAdes: A new genome assembly algorithm and its applications to single-cell sequencing. J. Comput. Biol. 19:455-477. https://doi.org/10.1089/cmb.2012.0021.
Bodilis, J., R. Calbrix, J. Guérillon, A. Mérieau, B. Pawlak, N. Orange, and S. Barray. 2004. Phylogenetic relationships between environmental and clinical isolates of Pseudomonas fluorescens and related species deduced from $16 \mathrm{~S}$ rRNA gene and OprF protein sequences. Syst. Appl. Microbiol. 27:93-108. https://doi.org/10 .1078/0723-2020-00253.

Bolger, A. M., M. Lohse, and B. Usadel. 2014. Trimmomatic: A flexible trimmer for Illumina sequence data. Bioinformatics 30:21142120. https://doi.org/10.1093/bioinformatics/btu170.

Botes, J., G. Williamson, V. Sinickas, and V. Gürtler. 2003. Genomic typing of Pseudomonas aeruginosa isolates by comparison of Riboprinting and PFGE: Correlation of experimental results with those predicted from the complete genome sequence of isolate PAO1. J. Microbiol. Methods 55:231-240. https://doi.org/10.1016/s0167 -7012(03)00156-8.

Caldera, L., L. Franzetti, E. Van Coillie, P. De Vos, P. Stragier, J. De Block, and M. Heyndrickx. 2016. Identification, enzymatic spoilage characterization and proteolytic activity quantification of Pseudomonas spp. isolated from different foods. Food Microbiol. 54:142-153. https://doi.org/10.1016/j.fm.2015.10.004.

Camacho, C., G. Coulouris, V. Avagyan, N. Ma, J. Papadopoulos, K. Bealer, and T. L. Madden. 2009. BLAST+: Architecture and applications. BMC Bioinformatics 10:421. https://doi.org/10.1186/ 1471-2105-10-421.

Carminati, D., B. Bonvini, L. Rossetti, M. Zago, F. Tidona, and G. Giraffa. 2019. Investigation on the presence of blue pigmentproducing Pseudomonas strains along a production line of fresh mozzarella cheese. Food Control 100:321-328. https://doi.org/10 .1016/j.foodcont.2019.02.009.

Carrascosa, C., R. Millán, J. R. Jaber, P. Lupiola, C. del RosarioQuintana, C. Mauricio, and E. Sanjuán. 2015. Blue pigment in fresh cheese produced by Pseudomonas fluorescens. Food Control 54:95-102. https://doi.org/10.1016/j.foodcont.2014.12.039.

Colantuono, A., P. D'Incecco, M. G. Fortina, V. Rosi, G. Ricci, and L. Pellegrino. 2020. Milk substrates influence proteolytic activity of Pseudomonas fluorescens strains. Food Control 111:107063. https: //doi.org/10.1016/j.foodcont.2019.107063.

Dabbah, R., W. A. Moats, and V. M. Edwards. 1971. Heat survivor curves of food-borne bacteria suspended in commercially sterilized whole milk. II. Bacteria other than Salmonellae. J. Dairy Sci. 54:1772-1779. https://doi.org/10.3168/jds.s0022-0302(71)86111-8.

de Vries, A., and B. D. Ripley. 2016. ggdendro: Create dendrograms and tree diagrams using "ggplot2." R package version 0.1-20. https: //CRAN.R-project.org/package=ggdendro.

del Olmo, A.. J. Calzada, and M. Nuñez. 2018. The blue discoloration of fresh cheeses: A worldwide defect associated to specific contamination by Pseudomonas fluorescens. Food Control 86:359-366. https://doi.org/10.1016/j.foodcont.2017.12.001.

Dixon, P. 2003. VEGAN, a package of R functions for community ecology. J. Veg. Sci. 14:927-930. https://doi.org/10.1111/j.1654-1103 .2003.tb02228.x.

Dogan, B., and K. J. Boor. 2003. Genetic diversity and spoilage potentials among Pseudomonas spp. isolated from fluid milk products and dairy processing plants. Appl. Environ. Microbiol. 69:130-138. https://doi.org/10.1128/AEM.69.1.130-138.2003.

Durak, M. Z., H. I. Fromm, J. R. Huck, R. N. Zadoks, and K. J. Boor. 2006. Development of molecular typing methods for Bacillus spp. and Paenibacillus spp. isolated from fluid milk products. J. Food Sci. 71:M50-M56. https://doi.org/10.1111/j.1365-2621.2006 .tb08907.x.

Edgar, R. C. 2004. MUSCLE: Multiple sequence alignment with high accuracy and high throughput. Nucleic Acids Res. 32:1792-1797. https://doi.org/10.1093/nar/gkh340.

Eneroth, A., S. Ahrné, and G. Molin. 2000. Contamination routes of Gram-negative spoilage bacteria in the production of pasteurised milk, evaluated by randomly amplified polymorphic DNA (RAPD). Int. Dairy J. 10:325-331. https://doi.org/10.1016/S0958 -6946(00)00055-8.

Fogel, G. B., C. R. Collins, J. Li, and C. F. Brunk. 1999. Prokaryotic genome size and SSU rDNA copy number: Estimation of micro- 
bial relative abundance from a mixed population. Microb. Ecol. 38:93-113. https://doi.org/10.1007/s002489900162.

Gardner, S. N., T. Slezak, and B. G. Hall. 2015. kSNP3.0: SNP detection and phylogenetic analysis of genomes without genome alignment or reference genome. Bioinformatics 31:2877-2878. https:// doi.org/10.1093/bioinformatics/btv271.

Griffiths, M., J. Phillips, and D. Muir. 1984. Post-pasteurization contamination - The major cause of failure of fresh dairy products. Hannah Res. 1984:77-87.

Gurevich, A., V. Saveliev, N. Vyahhi, and G. Tesler. 2013. QUAST: Quality assessment tool for genome assemblies. Bioinformatics 29:1072-1075. https://doi.org/10.1093/bioinformatics/btt086.

Hartmann, R. K., H. Y. Toschka, N. Ulbrich, and V. A. Erdmann. 1986. Genomic organization of rDNA in Pseudomonas aeruginosa. FEBS Lett. 195:187-193. https://doi.org/10.1016/0014-5793(86)80158-2.

Hayes, W., C. H. White, and M. A. Drake. 2002. Sensory aroma characteristics of milk spoilage by Pseudomonas species. J. Food Sci. 67:448-454. https://doi.org/10.1111/j.1365-2621.2002.tb11427.x.

Hesse, C., F. Schulz, C. T. Bull, B. T. Shaffer, Q. Yan, N. Shapiro, K. A. Hassan, N. Varghese, L. D. H. Elbourne, I. T. Paulsen, N. Kyrpides, T. Woyke, and J. E. Loper. 2018. Genome-based evolutionary history of Pseudomonas spp. Environ. Microbiol. 20:21422159. https://doi.org/10.1111/1462-2920.14130.

Hilario, E., T. R. Buckley, and J. M. Young. 2004. Improved resolution on the phylogenetic relationships among Pseudomonas by the combined analysis of atpD, carA, recA and $16 \mathrm{~S}$ rDNA. Antonie van Leeuwenhoek 86:51-64. https://doi.org/10.1023/B:ANTO .0000024910 .57117 .16 .

Johnson, J. K., S. M. Arduino, O. C. Stine, J. A. Johnson, and A. D. Harris. 2007. Multilocus sequence typing compared to pulsed-field gel electrophoresis for molecular typing of Pseudomonas aeruginosa. J. Clin. Microbiol. 45:3707-3712. https://doi.org/10.1128/ JCM.00560-07.

Kovac, J., R. A. Miller, L. M. Carroll, D. J. Kent, J. Jian, S. M. Beno, and M. Wiedmann. 2016. Production of hemolysin BL by Bacillus cereus group isolates of dairy origin is associated with wholegenome phylogenetic clade. BMC Genomics 17:581. https://doi .org/10.1186/s12864-016-2883-z.

Kumar, S., G. Stecher, M. Li, C. Knyaz, and K. Tamura. 2018. MEGA $\mathrm{X}$ : Molecular evolutionary genetics analysis across computing platforms. Mol. Biol. Evol. 35:1547-1549. https://doi.org/10.1093/ molbev/msy096.

Lagesen, K., P. Hallin, E. A. Rødland, H.-H. Stærfeldt, T. Rognes, and D. W. Ussery. 2007. RNAmmer: Consistent and rapid annotation of ribosomal RNA genes. Nucleic Acids Res. 35:3100-3108. https: //doi.org/10.1093/nar/gkm160.

Larsson, A. 2014. AliView: A fast and lightweight alignment viewer and editor for large datasets. Bioinformatics 30:3276-3278. https:/ /doi.org/10.1093/bioinformatics/btu531.

Liao, J., M. Wiedmann, and J. Kovac. 2017. Genetic stability and evolution of the sigB allele, used for Listeria sensu stricto subtyping and phylogenetic inference. Appl. Environ. Microbiol. 83:e0030617. https://doi.org/10.1128/AEM.00306-17.

Macaulay, D. M., R. Z. Hawirko, and N. James. 1963. Effect of pasteurization on survival of certain psychrophilic bacteria. Appl. Microbiol. 11:90-92. https://doi.org/10.1128/AEM.11.2.90-92.1963.

Martin, D. P., B. Murrell, A. Khoosal, and B. Muhire. 2017. Detecting and analyzing genetic recombination using RDP4. Pages 433-460 in Bioinformatics: Data, Sequence Analysis, and Evolution. Vol. I. J. M. Keith, ed. Springer, New York, NY.

Martin, N. H., S. C. Murphy, R. D. Ralyea, M. Wiedmann, and K. J. Boor. 2011. When cheese gets the blues: Pseudomonas fluorescens as the causative agent of cheese spoilage. J. Dairy Sci. 94:31763183. https://doi.org/10.3168/jds.2011-4312.

Masiello, S. N., N. H. Martin, A. Trmčić, M. Wiedmann, and K. J. Boor. 2016. Identification and characterization of psychrotolerant coliform bacteria isolated from pasteurized fluid milk. J. Dairy Sci. 99:130-140. https://doi.org/10.3168/jds.2015-9728.

Meng, L., H. Liu, L. Dong, N. Zheng, M. Xing, Y. Zhang, S. Zhao, and J. Wang. 2018. Identification and proteolytic activity quantification of Pseudomonas spp. isolated from different raw milks at stor- age temperatures. J. Dairy Sci. 101:2897-2905. https://doi.org/10 .3168/jds.2017-13617.

Meng, L., Y. Zhang, H. Liu, S. Zhao, J. Wang, and N. Zheng. 2017. Characterization of Pseudomonas spp. and associated proteolytic properties in raw milk stored at low temperatures. Front. Microbiol. 8:2158. https://doi.org/10.3389/fmicb.2017.02158.

Moore, E. R. B., M. Mau, A. Arnscheidt, E. C. Böttger, R. A. Hutson, M. D. Collins, Y. Van De Peer, R. De Wachter, and K. N. Timmis. 1996. The determination and comparison of the $16 \mathrm{~S}$ rRNA gene sequences of species of the genus Pseudomonas (sensu stricto) and estimation of the natural intrageneric relationships. Syst. Appl. Microbiol. 19:478-492. https://doi.org/10.1016/S0723 -2020(96)80021-X.

Nicodème, M., J.-P. Grill, G. Humbert, and J.-L. Gaillard. 2005. Extracellular protease activity of different Pseudomonas strains: Dependence of proteolytic activity on culture conditions. J. Appl. Microbiol. 99:641-648. https://doi.org/10.1111/j.1365-2672.2005 .02634.x.

Nightingale, K., L. Bovell, A. Grajczyk, and M. Wiedmann. 2007. Combined $\operatorname{sig} B$ allelic typing and multiplex PCR provide improved discriminatory power and reliability for Listeria monocytogenes molecular serotyping. J. Microbiol. Methods 68:52-59. https://doi .org/10.1016/j.mimet.2006.06.005.

Page, A. J., B. Taylor, and J. A. Keane. 2016. Multilocus sequence typing by blast from de novo assemblies against PubMLST. J. Open Source Softw. 1:118. https://doi.org/10.21105/joss.00118.

Parks, D. H., M. Chuvochina, D. W. Waite, C. Rinke, A. Skarshewski, P.-A. Chaumeil, and P. Hugenholtz. 2018. A standardized bacterial taxonomy based on genome phylogeny substantially revises the tree of life. Nat. Biotechnol. 36:996-1004. https://doi.org/10 $.1038 /$ nbt. 4229 .

Porteous, L. A., F. Widmer, and R. J. Seidler. 2002. Multiple enzyme restriction fragment length polymorphism analysis for high resolution distinction of Pseudomonas (sensu stricto) 16S rRNA genes. J. Microbiol. Methods 51:337-348. https://doi.org/10.1016/s0167 -7012(02)00108-2.

R Core Team. 2019. R: A Language and Environment for Statistical Computing. R Foundation for Statistical Computing, Vienna, Austria.

Ralyea, R. D., M. Wiedmann, and K. J. Boor. 1998. Bacterial tracking in a dairy production system using phenotypic and ribotyping methods. J. Food Prot. 61:1336-1340. https://doi.org/10.4315/ 0362-028x-61.10.1336.

Reichler, S. J., N. H. Martin, R. L. Evanowski, J. Kovac, M. Wiedmann, and R. H. Orsi. 2019. A century of gray: A genomic locus found in 2 distinct Pseudomonas spp. is associated with historical and contemporary color defects in dairy products worldwide. J. Dairy Sci. 102:5979-6000. https://doi.org/10.3168/jds.2018-16192.

Reichler, S. J., S. I. Murphy, T. Erickson, N. H. Martin, K. J. Boor, and M. Wiedmann. 2020a. Employee training and updated chemical sanitation may be insufficient to ameliorate spoilage due to postpasteurization contamination in HTST fluid milk processing facilities. J. Dairy Sci. 103:7569-7584. https://doi.org/10.3168/jds .2020-18186.

Reichler, S. J., S. I. Murphy, N. H. Martin, and M. Wiedmann. 2020b. Supplemental data for: Identification, subtyping, and tracking of dairy spoilage-associated Pseudomonas by sequencing the ileS gene. Cornell University eCommons Repository. https://doi.org/ $10.7298 /$ rekh-4w25.

Reichler, S. J., A. Trmčić, N. H. Martin, K. J. Boor, and M. Wiedmann. 2018. Pseudomonas fluorescens group bacterial strains are responsible for repeat and sporadic postpasteurization contamination and reduced fluid milk shelf life. J. Dairy Sci. 101:7780-7800. https://doi.org/10.3168/jds.2018-14438.

Remenant, B., E. Jaffrès, X. Dousset, M.-F. Pilet, and M. Zagorec. 2015. Bacterial spoilers of food: Behavior, fitness and functional properties. Food Microbiol. 45:45-53. https://doi.org/10.1016/j .fm.2014.03.009.

Rodrigues, M. X., S. F. Lima, S. G. Canniatti-Brazaca, and R. C. Bicalho. 2017. The microbiome of bulk tank milk: Characterization 
and associations with somatic cell count and bacterial count. J. Dairy Sci. 100:2536-2552. https://doi.org/10.3168/jds.2016-11540.

Rozas, J., A. Ferrer-Mata, J. C. Sánchez-DelBarrio, S. Guirao-Rico, P. Librado, S. E. Ramos-Onsins, and A. Sánchez-Gracia. 2017. DnaSP 6: DNA sequence polymorphism analysis of large data sets. Mol. Biol. Evol. 34:3299-3302. https://doi.org/10.1093/molbev/ $\operatorname{msx} 248$.

Sarkar, S. F., and D. S. Guttman. 2004. Evolution of the core genome of Pseudomonas syringae, a highly clonal, endemic plant pathogen. Appl. Environ. Microbiol. 70:1999-2012. https://doi.org/10.1128/ AEM.70.4.1999-2012.2004.

Schliep, K. P. 2011. phangorn: Phylogenetic analysis in R. Bioinformatics 27:592-593. https://doi.org/10.1093/bioinformatics/btq706.

Schröder, M. J. 1984. Origins and levels of post pasteurization contamination of milk in the dairy and their effects on keeping quality. J. Dairy Res. 51:59-67. https://doi.org/10.1017/s0022029900023323.

Stamatakis, A. 2014. RAxML version 8: A tool for phylogenetic analysis and post-analysis of large phylogenies. Bioinformatics 30:13121313. https://doi.org/10.1093/bioinformatics/btu033.

Stessl, B., I. Rückerl, and M. Wagner. 2014. Multilocus sequence typing (MLST) of Listeria monocytogenes. Pages 73-83 in Listeria monocytogenes: Methods and Protocols. K. Jordan, E. M. Fox, and M. Wagner, ed. Springer, New York, NY.

Sunga, F. C. A., D. R. Heldman, and T. I. Hedrick. 1970. Microorganisms from arms and hands of dairy plant workers. J. Milk Food Technol. 33:178-181. https://doi.org/10.4315/0022-2747-33.5.178.

Trmčić, A., N. H. Martin, K. J. Boor, and M. Wiedmann. 2015. A standard bacterial isolate set for research on contemporary dairy spoilage. J. Dairy Sci. 98:5806-5817. https://doi.org/10.3168/jds .2015-9490

Truong, D. T., E. A. Franzosa, T. L. Tickle, M. Scholz, G. Weingart, E. Pasolli, A. Tett, C. Huttenhower, and N. Segata. 2015. MetaPhlAn2 for enhanced metagenomic taxonomic profiling. Nat. Methods 12:902-903. https://doi.org/10.1038/nmeth.3589.

van Belkum, A., L. B. Soriaga, M. C. LaFave, S. Akella, J.-B. Veyrieras, E. M. Barbu, D. Shortridge, B. Blanc, G. Hannum, G. Zambardi, K. Miller, M. C. Enright, N. Mugnier, D. Brami, S. Schicklin, M. Felderman, A. S. Schwartz, T. H. Richardson, T. C. Peterson, B. Hubby, and K. C. Cady. 2015. Phylogenetic distribution of CRISPR-Cas systems in antibiotic-resistant Pseudomonas aeruginosa. MBio 6:e01796-15. https://doi.org/10.1128/mBio $.01796-15$.
Weckbach, L. S., and B. E. Langlois. 1977. Effect of heat treatments on survival and growth of a psychrotroph and on nitrogen fractions in milk. J. Food Prot. 40:857-862. https://doi.org/10.4315/0362 $-028 \mathrm{X}-40.12 .857$.

Werle, E., C. Schneider, M. Renner, M. Völker, and W. Fiehn. 1994. Convenient single-step, one tube purification of PCR products for direct sequencing. Nucleic Acids Res. 22:4354-4355. https://doi .org/10.1093/nar/22.20.4354.

Wiedmann, M., D. Weilmeier, S. S. Dineen, R. Ralyea, and K. J. Boor. 2000. Molecular and phenotypic characterization of Pseudomonas spp. isolated from milk. Appl. Environ. Microbiol. 66:2085-2095. https://doi.org/10.1128/aem.66.5.2085-2095.2000.

Woods, R. G., M. Burger, C.-A. Beven, and I. R. Beacham. 2001. The aprX-lipA operon of Pseudomonas fluorescens B52: A molecular analysis of metalloprotease and lipase production. Microbiology 147:345-354. https://doi.org/10.1099/00221287-147-2-345.

Worm, J., L. E. Jensen, T. S. Hansen, M. Søndergaard, and O. Nybroe. 2000. Interactions between proteolytic and non-proteolytic Pseudomonas fluorescens affect protein degradation in a model community. FEMS Microbiol. Ecol. 32:103-109. https://doi.org/ 10.1111/j.1574-6941.2000.tb00704.x.

Yamamoto, S., H. Kasai, D. L. Arnold, R. W. Jackson, A. Vivian, and S. Harayama. 2000. Phylogeny of the genus Pseudomonas: Intrageneric structure reconstructed from the nucleotide sequences of gyrB and rpoD genes. Microbiology 146:2385-2394. https://doi .org/10.1099/00221287-146-10-2385.

Zhang, C., E. Bijl, B. Svensson, and K. Hettinga. 2019. The extracellular protease AprX from Pseudomonas and its spoilage potential for UHT milk: A review. Compr. Rev. Food Sci. Food Saf. 18:834-852. https://doi.org/10.1111/1541-4337.12452.

\section{ORCIDS}

S. J. Reichler () https://orcid.org/0000-0001-5288-2517

S. I. Murphy ๑ https://orcid.org/0000-0001-9092-0625

N. H. Martin (ํ) https://orcid.org/0000-0003-1704-0634

M. Wiedmann (ํ) https://orcid.org/0000-0002-4168-5662 